\title{
Effect of natalizumab on disease progression in secondary progressive multiple sclerosis (ASCEND): a phase 3, randomised, double-blind, placebo-controlled trial with an open-label extension
}

\author{
Raju Kapoor, Pei-Ran Ho, Nolan Campbell, Ih Chang, Aaron Deykin, Fiona Forrestal, Nisha Lucas, Bei Yu, Douglas L Arnold, Mark S Freedman, \\ Myla D Goldman, Hans-Peter Hartung, Eva Kubala Haurdová, Douglas Jeffery, Aaron Miller, Finn Sellebjerg, Diego Cadavid, Dan Mikol, Deborah Steiner, \\ on behalf of the ASCEND investigators*
}

\section{Summary}

Background Although several disease-modifying treatments are available for relapsing multiple sclerosis, treatment effects have been more modest in progressive multiple sclerosis and have been observed particularly in actively relapsing subgroups or those with lesion activity on imaging. We sought to assess whether natalizumab slows disease progression in secondary progressive multiple sclerosis, independent of relapses.

Methods ASCEND was a phase 3, randomised, double-blind, placebo-controlled trial (part 1) with an optional 2 year open-label extension (part 2). Enrolled patients aged 18-58 years were natalizumab-naive and had secondary progressive multiple sclerosis for 2 years or more, disability progression unrelated to relapses in the previous year, and Expanded Disability Status Scale (EDSS) scores of 3.0-6.5. In part 1, patients from 163 sites in 17 countries were randomly assigned (1:1) to receive $300 \mathrm{mg}$ intravenous natalizumab or placebo every 4 weeks for 2 years. Patients were stratified by site and by EDSS score (3.0-5.5 vs 6.0-6.5). Patients completing part 1 could enrol in part 2, in which all patients received natalizumab every 4 weeks until the end of the study. Throughout both parts, patients and staff were masked to the treatment received in part 1 . The primary outcome in part 1 was the proportion of patients with sustained disability progression, assessed by one or more of three measures: the EDSS, Timed 25-Foot Walk (T25FW), and 9-Hole Peg Test (9HPT). The primary outcome in part 2 was the incidence of adverse events and serious adverse events. Efficacy and safety analyses were done in the intention-to-treat population. This trial is registered with ClinicalTrials.gov, number NCT01416181.

Findings Between Sept 13, 2011, and July 16, 2015, 889 patients were randomly assigned ( $\mathrm{n}=440$ to the natalizumab group, $n=449$ to the placebo group). In part 1, 195 (44\%) of 439 natalizumab-treated patients and 214 (48\%) of 448 placebo-treated patients had confirmed disability progression (odds ratio [OR] 0.86; 95\% CI 0.66-1.13; $\mathrm{p}=\mathbf{0} \cdot 287$ ). No treatment effect was observed on the EDSS (OR 1.06, 95\% CI $0 \cdot 74-1 \cdot 53$; nominal $\mathrm{p}=0.753$ ) or the T25FW $(0 \cdot 98,0 \cdot 74-1 \cdot 30$; nominal $\mathrm{p}=0.914)$ components of the primary outcome. However, natalizumab treatment reduced 9HPT progression (OR $0.56,95 \%$ CI $0 \cdot 40-0 \cdot 80$; nominal $\mathrm{p}=0 \cdot 001$ ). In part 1,100 (22\%) placebo-treated and $90(20 \%)$ natalizumab-treated patients had serious adverse events. In part 2, 291 natalizumab-continuing patients and 274 natalizumab-naive patients received natalizumab (median follow-up 160 weeks [range 108-221]). Serious adverse events occurred in 39 (13\%) patients continuing natalizumab and in 24 (9\%) patients initiating natalizumab. Two deaths occurred in part 1 , neither of which was considered related to study treatment. No progressive multifocal leukoencephalopathy occurred.

Interpretation Natalizumab treatment for secondary progressive multiple sclerosis did not reduce progression on the primary multicomponent disability endpoint in part 1, but it did reduce progression on its upper-limb component. Longer-term trials are needed to assess whether treatment of secondary progressive multiple sclerosis might produce benefits on additional disability components.

Funding Biogen.

\section{Introduction}

Patients with multiple sclerosis usually present with neurological relapses and the disorder evolves to accumulate gradual disability with or without superimposed relapses in the secondary progressive phase. ${ }^{1}$ Disease-modifying treatments are increasingly available for relapsing multiple sclerosis; however, despite numerous studies, ${ }^{2-12}$ benefits in trials of progressive multiple sclerosis are modest and mostly target actively relapsing subgroups or those with lesion activity on imaging. Although recent results have suggested a treatment effect of siponimod in secondary progressive multiple scleroisis, ${ }^{13}$ only mitoxantrone has received approval for delaying disability in secondary progressive
Lancet Neurol 2018; 17: 405-15 Published Online March 12, 2018 http://dx.doi.org/10.1016/ S1474-4422(18)30069-3

See Comment page 384 *ASCEND investigators are listed at the end of the Article

National Hospital for Neurology and Neurosurgery, University College London Hospitals NHS Foundation Trust, London, UK (R Kapoor FRCP); Biogen, Cambridge, MA, USA (P-R Ho MD, N Campbell PhD IChang PhD, A Deykin MD, F Forrestal MS, N Lucas MS, B Yu MD, D Steiner MD D Cadavid MD, D Mikol MD); Montreal Neurological Institute, Montreal, QC, Canada (D L Arnold MD); NeuroRx Research, Montreal, QC, Canada (D L Arnold); University of Ottawa, Ottawa Hospital Research Institute, Ottawa, ON, Canada (M S Freedman MD); University of Virginia, Charlottesville, VA, USA (M D Goldman MD); Department of Neurology, Medical Faculty, Heinrich-Heine University, Düsseldorf, Germany (Prof H-P Hartung MD); First Faculty of Medicine and General University Hospital Charles University, Prague, Czech Republic (Prof E K Havrdová MD); Piedmont HealthCare, Mooresville, NC, USA (D Jeffery MD); Icahn School of Medicine at Mount Sinai, New York, NY, USA (A Miller MD); and Danish Multiple Sclerosis Center Rigshospitalet, University of Copenhagen, Copenhagen, Denmark (Prof F Sellebjerg MD) 


\author{
Correspondence to: \\ Dr Raju Kapoor, National \\ Hospital for Neurology and \\ Neurosurgery, University College \\ London Hospitals NHS \\ Foundation Trust, London \\ WC1N 3BG, UK \\ r.kapoor@nhs.net
}

Research in context

\section{Evidence before this study}

We searched PubMed for English language articles published from Jan 1, 1970, to Sept 1, 2017, using the keyword "secondary progressive multiple sclerosis", on clinical trials of diseasemodifying treatments in secondary progressive multiple sclerosis and studies of natalizumab and relevant outcome measures. All clinical trials were included. Despite numerous trials, positive treatment effects on disability progression were noted only in phase 2 trials of simvastatin and methotrexate and in phase 3 trials of interferon beta- $1 \mathrm{~b}$ and mitoxantrone. Positive results on disability progression in a phase 3 trial of siponimod were recently presented in a conference abstract. In a phase 2 proof-of-concept study of natalizumab in progressive forms of multiple sclerosis, treatment reduced markers of intrathecal inflammation and tissue damage, suggesting that natalizumab might have therapeutic efficacy in progressive forms of multiple sclerosis. Retrospective analysis of outcomes in two early-phase studies of natalizumab in secondary progressive multiple sclerosis indicated non-significant improvement in ambulation. For assessment of treatment effects in progressive multiple sclerosis, the Expanded Disability Status Scale (EDSS) was found to have poor responsiveness in patients with high baseline disability scores, and detection of disability progression in secondary progressive multiple sclerosis was improved with a multicomponent endpoint consisting of the EDSS, the Timed 25-Foot Walk, and the 9-Hole Peg Test. This multicomponent endpoint might represent a better measure to detect disability progression in secondary progressive multiple sclerosis by providing similar power with approximately half the sample size.

\section{Added value of this study}

The ASCEND study is, to our knowledge, the first phase 3 trial to assess the efficacy of natalizumab on sustained disability progression in mostly non-relapsing patients with secondary progressive multiple sclerosis, a population with a high unmet need for disease-modifying treatments. The multicomponent primary outcome used in our study could measure disability progression more sensitively than the EDSS alone. Although natalizumab treatment did not affect progression of the multicomponent primary outcome in the randomised controlled phase of the study, we found evidence that the upper-limb component of disability did respond to treatment and that beneficial effects on the ambulatory components were evident with more prolonged therapy (beyond 2 years) in the open-label extension phase, in which masking of patients and staff to treatment allocation in the placebo-controlled phase was maintained.

\section{Implications of all the available evidence}

Although natalizumab did not affect progression of the primary disability outcome in the placebo-controlled phase of ASCEND, it consistently had a positive effect on progression of upper-limb disability in part 1 of the study and in those patients who continued to receive the treatment in part 2. Results from the open-label extension raise the possibility that a positive effect on the ambulatory components of the primary disability outcome requires more than 2 years of treatment. However, it should be noted that secondary outcomes in part 1 did not show a treatment effect and the effects observed in part 2 are based on nominal significance. Although the mechanisms of the positive effects observed in this study require further consideration, they raise important issues for trial designs for secondary progressive multiple sclerosis: future trials might require treatment phases lasting 3 years or more, and both investigators and regulators might need to anticipate the possibility that benefits could be restricted to specific but nevertheless key domains such as upper-limb ability. multiple sclerosis, and its use is restricted by substantial risks of cardiotoxicity and leukaemia. Hence, an important unmet need exists for therapies in secondary progressive multiple sclerosis that prevent disability progression unrelated to relapse.

Treatments for relapsing multiple sclerosis generally act on the peripheral immune response. ${ }^{14}$ Their limited effects on progressive disability suggest that the mechanisms of tissue injury are diverse in progressive multiple sclerosis. Particular interest has emerged in the intrathecal immune response, which is shielded from peripherally acting agents behind the blood-brain barrier. ${ }^{14}$ Natalizumab, a recombinant humanised monoclonal antibody against $\alpha 4$ integrin (very late antigen-4), is highly effective in relapsing-remitting multiple sclerosis ${ }^{15}$ and appears to suppress intrathecal inflammation. ${ }^{14}$ Natalizumab inhibits leucocyte transmigration across the blood-brain barrier, interferes with chemokine-mediated inflammatory-cell recruitment into the CNS, and disrupts the production of molecules required to sustain intrathecal inflammation..$^{16-18}$ Results from early phase trials in progressive multiple sclerosis show that natalizumab treatment suppresses CSF markers of inflammation and neurodegeneration, including concentrations of osteopontin and the B-cell chemokine CXCL13, ${ }^{14}$ and suggest that treatment also improves ambulation and upper-limb function. ${ }^{19-21}$

On the basis of these considerations, we aimed to investigate whether treatment with natalizumab is safe and slows the accumulation of disability unrelated to relapse in patients with secondary progressive multiple sclerosis.

\section{Methods \\ Study design}

The ASCEND study was an international, multicentre, phase 3 clinical trial done in two parts. In the randomised, double-blind, parallel-group, placebo-controlled phase 
(part 1), patients with secondary progressive multiple sclerosis from 163 sites in 17 countries received natalizumab or placebo and underwent scheduled study assessments for up to 96 weeks. The 17 countries were Belgium, Canada, the Czech Republic, Denmark, Finland, France, Germany, Ireland, Israel, Italy, the Netherlands, Poland, Russia, Spain, Sweden, the UK, and the USA.

In the prespecified, optional open-label extension phase for safety and efficacy (part 2), all patients who completed part 1 could receive natalizumab until the end of the study. Patients who did not enrol in part 2 stopped blinded study treatment at week 96 and returned to the study site for a follow-up visit at week 108. Patients who opted to enrol in part 2 continued to receive blinded study treatment every 4 weeks between enrolment into part 2 (week 96) and the start of open-label treatment at week 108. In the protocol, the overall end of the study was defined as the last patient's final visit for collection of data in part 2 . However, part 2 was terminated by the after the results of part 1 were released, before completion of year 4 of the study, because a significant effect on the primary multicomponent endpoint in part 1 was not observed. Because of the premature trial termination, the efficacy analysis was based on data collected up to week 156 .

The ASCEND study was done in accordance with the Declaration of Helsinki, the International Conference on Harmonisation, and good clinical practice guidelines. Approval for the study protocol, including any amendments, was granted by each centre's ethics committee, and all patients provided written informed consent before initiating any study-related activities.

An independent drug safety monitoring committee reviewed interim unblinded safety data for part 1 of the study. This committee met quarterly to review safety data and to advise on changes in study design. This committee was also charged with determining whether the study should be stopped or amended for reasons other than safety.

\section{Participants}

Natalizumab-naive patients aged $18-58$ years were eligible for enrolment in part 1 of the ASCEND study if they had onset of secondary progressive multiple sclerosis 2 or more years before enrolment, an Expanded Disability Status Scale (EDSS) score of 3.0-6.5 (inclusive), a Multiple Sclerosis Severity Score of 4 or more, and disability progression not related to clinical relapses during the year before enrolment, as assessed by clinical historical findings with a standardised form (appendix). Unlike previous secondary progressive multiple sclerosis trials that enrolled patients with recent clinical relapses, ${ }^{2,10,11}$ ASCEND excluded patients who had a clinical relapse up to 3 months before randomisation (to prevent recent relapses from influencing the baseline assessment of disability). A full list of part 1 inclusion and exclusion criteria is included in the appendix.
For inclusion in part 2, eligible patients were required to have participated in part 1 and to have completed all part 1 examinations and efficacy assessments before receiving the first open-label dose at week 108 in part 2. Patients were excluded from part 2 if they had discontinued study treatment, received less than 20 infusions, or missed two or more consecutive infusions in part 1.

\section{Randomisation and masking}

In part 1 of ASCEND, eligible patients were randomly assigned (1:1) to receive natalizumab or placebo of identical appearance. Patients were stratified by site and by EDSS score $(3 \cdot 0-5 \cdot 5$ vs $6 \cdot 0-6 \cdot 5)$. Patients and study staff were masked to treatment assignments in part 1 , and patients enrolling in part 2 also received blinded study treatment during weeks 100 and 104. Patients in part 2 received open-label treatment starting in week 108. Both patients and study staff remained masked to the treatment assignments in part 2. Only the pharmacists preparing the infusion and the pharmacy study monitors were not masked to the study treatment, which was stored in a secure location and accounted for by the investigator. Patients were randomly assigned by an interactive voice/web response system (IXRS, Bracket Global LLC, San Francisco, CA, USA).

\section{Procedures}

In part 1 , patients received $300 \mathrm{mg}$ intravenous natalizumab or placebo every 4 weeks for 2 years. In the open-label extension phase in part 2, all patients received $300 \mathrm{mg}$ intravenous natalizumab every 4 weeks until the end of the study. In part 1, EDSS, Timed 25-Foot Walk (T25FW), and 9-Hole Peg Test (9HPT) assessments were done at baseline and every 12 weeks up to week 108 . Additional details of T25FW, 9HPT, and MRI assessments are provided in the appendix. During part 2, EDSS, T25FW, and 9HPT assessments were done at week 156 . The last progression events that could be confirmed in part 1 started at week 84, with final confirmation at week 108 (appendix). The additional progression events ascertained in part 2 were possible progression starting at weeks 96 and 108, when patients who entered part 2 were still being treated with their blinded therapy assignments from part 1 . Because of the premature trial termination, events at weeks 96 and 108 were confirmed at week 156 for patients in part 2, during which all patients were treated with open-label natalizumab.

Patients underwent a blood test for presence of anti-JC virus (JCV) antibodies at baseline and every 24 weeks during parts 1 and 2 . As per the study protocol, the principal investigator received anti-JCV antibody results throughout the study. Investigators were informed of a patient's anti-JCV antibody status before enrolment in part 2 and at 24 week intervals thereafter.

Treatment-emergent adverse events and serious adverse events were recorded in the safety population during parts 1 and 2. Over the course of parts 1 and 2 (every 12 weeks up 
to week 108 and at week 156), the treating neurologist completed the equivalent of a regular clinic visit. An independent neurology evaluation committee (INEC) confirmed whether patients had a protocol-defined

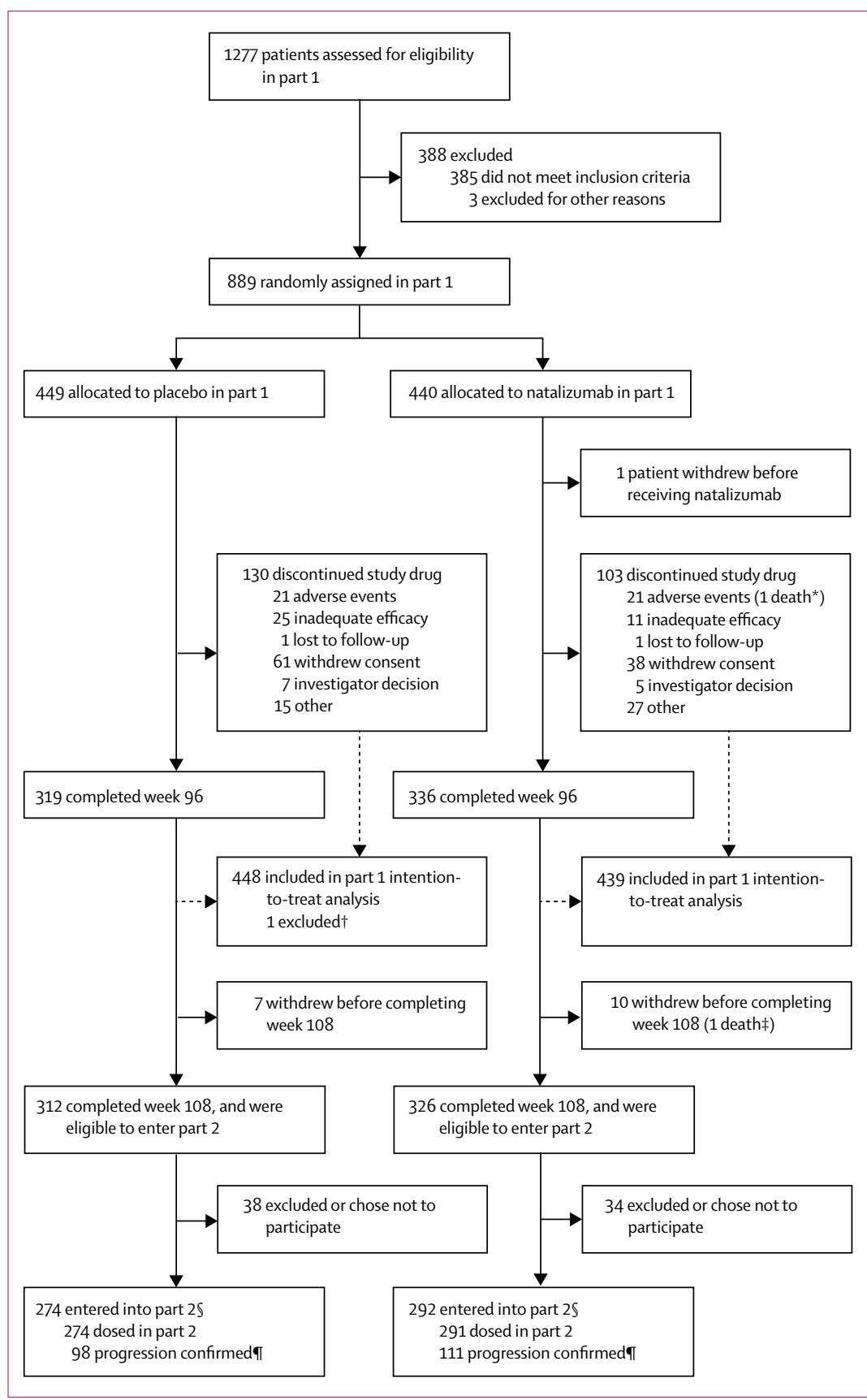

Figure 1: Trial profile

* One patient diagnosed with lung cancer (unrelated to study treatment) discontinued because of this serious adverse event. Follow-up revealed that this serious adverse event resulted in death after discontinuation. †One patient received the first dose of treatment at week 28 and was therefore not included in the part 1 intention-totreat population. ¥Death of one patient, due to septic shock with multiple organ failure (unrelated to study treatment), occurred after completion of 96 weeks of treatment but before completion of 108 weeks in the study. \$Part 2 was terminated by the funder after the results of part 1 were released. $₫ A$ t week 156, progression was confirmed for weeks 96 and 108 in 209 patients. relapse. All reviews were done without knowledge of the patient's treatment assignment and without an MRI scan.

\section{Outcomes}

The primary outcome in part 1 was based on a multicomponent measure of sustained disability progression comprising the EDSS, T25FW, and 9HPT, and was designed to provide a more sensitive and specific measure of disease worsening in secondary progressive multiple sclerosis than EDSS alone. ${ }^{22}$ The primary endpoint was the proportion of patients with confirmed disability progression over the 96 week treatment period, with progression defined as meeting one or more of the following three criteria: an increase of 1.0 points or more from a baseline score of 5.5 or lower or an increase of 0.5 points or more from a baseline score of 6.0 or higher on the EDSS; an increase of $20 \%$ or more from baseline on the T25FW; or an increase of $20 \%$ or more from baseline (on either hand) on the 9HPT. Progression was confirmed at a subsequent visit 6 or more months after the start of a possible progression and at the end of the trial. To minimise the possibility of capturing disability progression due to clinical relapses, included disability progression events could not start or be confirmed 74 or fewer days following onset of an INEC-confirmed clinical relapse. The primary endpoint in part 2 was the incidence of adverse events and serious adverse events in patients with secondary progressive multiple sclerosis.

In part 1, secondary endpoints included the proportion of patients with consistent improvement in T25FW, change in patient-reported ambulatory status as measured by the 12-item Multiple Sclerosis Walking Scale (MSWS-12), change in patient-reported manual ability based on the ABILHAND questionnaire, ${ }^{23}$ the effect of natalizumab on patient-reported quality of life with the Multiple Sclerosis Impact Scale-29 (MSIS-29) physical score, change in whole brain volume between week 24 and week 96, and the proportion of patients with disability progression measured by individual physical EDSS functional system scores. Part 2 secondary endpoints included the proportion of patients with disability progression on the multicomponent endpoint confirmed during the additional follow-up time in part 2; change in T25FW, 9HPT, and EDSS from part 1 baseline to week 156; change in whole brain volume from part 1 week 24 to week 156; change in grey matter brain volume from part 1 baseline to week 156; change in number of T2 lesions from part 1 baseline to week 156; change in 6-Minute Walk Test, MSIS-29 Physical, and Symbol Digit Modalities Test (SDMT) scores from part 1 baseline to week 156; and change in Work Productivity and Activity Impairment Questionnaire score for multiple sclerosis from part 2 baseline (week 96) to week 156.

For the SDMT, confirmed progressors were defined as those showing a decrease of 4 points or more from part 1 baseline. Change from baseline over time for T25FW and 9HPT (each hand) was assessed for 


\begin{tabular}{|c|c|c|c|c|}
\hline & \multicolumn{2}{|l|}{ Part 1} & \multicolumn{2}{|l|}{ Part 2} \\
\hline & $\begin{array}{l}\text { Placebo } \\
(\mathrm{n}=448)\end{array}$ & $\begin{array}{l}\text { Natalizumab } 300 \mathrm{mg} \\
(\mathrm{n}=439)\end{array}$ & $\begin{array}{l}\text { Initiating natalizumab } \\
(\mathrm{n}=274)\end{array}$ & $\begin{array}{l}\text { Continuing natalizumab } \\
(\mathrm{n}=291)\end{array}$ \\
\hline Age, years & $47 \cdot 2(7 \cdot 8)$ & $47 \cdot 3(7.4)$ & $47.5(7.5)$ & $47 \cdot 2(7 \cdot 3)$ \\
\hline Women & $280(63 \%)$ & $270(62 \%)$ & $177(65 \%)$ & $180(62 \%)$ \\
\hline Men & $168(37 \%)$ & $169(38 \%)$ & $97(35 \%)$ & $111(38 \%)$ \\
\hline Years since first multiple sclerosis symptoms & $16 \cdot 2(7 \cdot 8)$ & $16 \cdot 8(7 \cdot 6)$ & $16 \cdot 5(7 \cdot 9)$ & $16.6(7 \cdot 4)$ \\
\hline $\begin{array}{l}\text { Years since secondary progressive multiple } \\
\text { sclerosis diagnosis }\end{array}$ & $4.9(3 \cdot 7)$ & $4 \cdot 7(3 \cdot 0)$ & $4 \cdot 6(3 \cdot 3)$ & $4 \cdot 8(2 \cdot 9)$ \\
\hline Years since most recent relapse before study & $4.8(4 \cdot 4)^{*}$ & $4 \cdot 7(4 \cdot 1)^{*}$ & $4 \cdot 8(4 \cdot 4)$ & $4 \cdot 8(4 \cdot 3)$ \\
\hline Median EDSS score & $6 \cdot 0(5 \cdot 0-6 \cdot 5)$ & $6 \cdot 0(5 \cdot 0-6 \cdot 5)$ & $6 \cdot 0(5 \cdot 0-6 \cdot 5)$ & $6 \cdot 0(5 \cdot 0-6 \cdot 5)$ \\
\hline Patients with EDSS score of 3.0-5.5 & $166(37 \%)$ & $165(38 \%)$ & $108(39 \%)$ & $111(38 \%)$ \\
\hline Patients with EDSS score of $6 \cdot 0-6.5$ & $282(63 \%)$ & $274(62 \%)$ & $166(61 \%)$ & $180(62 \%)$ \\
\hline Median T25FW, s & $11 \cdot 2(7 \cdot 9-16 \cdot 8)$ & $11 \cdot 2(7 \cdot 9-17 \cdot 5)$ & $11 \cdot 1(7 \cdot 6-17 \cdot 0)$ & $11 \cdot 0(7 \cdot 8-16 \cdot 7)$ \\
\hline \multicolumn{5}{|l|}{ Median 9HPT, $s$} \\
\hline Dominant hand & $28.8(23.8-36.6)$ & $28 \cdot 2(23 \cdot 5-36 \cdot 4)$ & $28.6(23 \cdot 2-36 \cdot 7)$ & $27.8(23.6-36 \cdot 4)$ \\
\hline Non-dominant hand & $29 \cdot 6(24 \cdot 7-40 \cdot 2)$ & $29 \cdot 6(25 \cdot 0-38 \cdot 5)$ & $29.5(24 \cdot 1-40 \cdot 8)$ & $29 \cdot 5(24 \cdot 8-39 \cdot 0)$ \\
\hline Patients with Gd+ lesions & $96(22 \%) \dagger$ & $114(26 \%) \dagger$ & $54(20 \%)$ & $77(26 \%)$ \\
\hline T2 lesion volume, $\mathrm{cm}^{3}$ & $16 \cdot 2(16 \cdot 4) \ddagger$ & $17 \cdot 4(17 \cdot 6) \ddagger$ & $16 \cdot 3(16 \cdot 4)$ & $17 \cdot 7(18 \cdot 5)$ \\
\hline Normalised whole brain volume, $\mathrm{cm}^{3}$ & $1425 \cdot 8(83 \cdot 1) \S$ & $1420 \cdot 9(82.8) \S$ & $1431 \cdot 2(82 \cdot 0)$ & $1425 \cdot 3(80 \cdot 3)$ \\
\hline $\begin{array}{l}\text { Data are } n(\%) \text {, mean (SD), or median (IQR). EDSS=E } \\
\text { for placebo; } n=431 \text { for natalizumab. } \hbar n=446 \text { for pla }\end{array}$ & $\begin{array}{l}\text { ded Disability Status } \\
; \mathrm{n}=438 \text { for natalizum }\end{array}$ & $\begin{array}{l}=\text { gadolinium enhanci } \\
7 \text { for placebo; } n=437\end{array}$ & $\begin{array}{l}\text { W=Timed 25-Foot Walk. } \\
\text { izumab. } 5 n=444 \text { for plac }\end{array}$ & $\begin{array}{l}=9-\text { Hole Peg Test. }{ }^{*} \mathrm{n}=430 \\
=436 \text { for natalizumab. }\end{array}$ \\
\hline
\end{tabular}

confirmed T25FW/9HPT progressors versus nonprogressors. Additionally, a sensitivity analysis of confirmed progression on $\mathrm{T} 25 \mathrm{FW}$ at thresholds other than $20 \%(25 \%, 30 \%, 40 \%$, and $50 \%)$ was done. Annualised relapse rate (ARR) was assessed, as was change from baseline over time for gadoliniumenhancing $(\mathrm{Gd}+)$ lesions. The number and volume of new and enlarging T2 lesions were assessed throughout the study. Finally, disability outcomes were assessed for subgroups with or without baseline Gd+ lesions and relapses in the 1-2 years before entering the study.

\section{Statistical analysis}

Baseline data were described with summary statistics. Treatment comparisons for efficacy endpoints were assessed with two-sided tests at a significance level of $0 \cdot 05$. A closed testing procedure was used to adjust for multiple secondary endpoints. All part 1 efficacy analyses were done in the part 1 intention-to-treat population, defined as all randomly assigned patients treated at baseline. The part 2 intention-to-treat population comprised all patients who were randomly assigned in part 1 and received one or more infusions of the study treatment in part 2. The primary endpoint of the percentage of patients with confirmed progression was analysed by use of logistic regression with baseline EDSS $(\leq 5 \cdot 5$ or $\geq 6 \cdot 0$ ), T25FW, and 9 HPT of each hand as covariates. A sensitivity analysis of the primary endpoint based on time to confirmed progression was done with a Cox proportional-hazards model with baseline EDSS $(\leq 5 \cdot 5$ or $\geq 6 \cdot 0)$, T25FW, and 9 HPT of each hand as covariates. Time to confirmed progression was defined as the time from the first infusion of study treatment to the first onset of possible progression that was subsequently confirmed. For secondary and exploratory endpoints, analysis of covariance and mixed-effects models for repeated measures were used to analyse continuous outcomes, with baseline measurement and baseline EDSS $(\leq 5 \cdot 5$ or $\geq 6 \cdot 0)$ as covariates, and logistic regression was used for dichotomous outcomes. For all exploratory analyses, all $\mathrm{p}$ values cited should be considered nominal unless otherwise stated.

The sample size was based on an assumed rate of confirmed disability progression over the 2 year period of $28 \%$ for the natalizumab group versus $40 \%$ for the placebo group -a $30 \%$ reduction. We estimated that 856 patients (428 per group) would provide $90 \%$ power at the 0.05 level of significance with a two-sided $\chi^{2}$ test, assuming a $20 \%$ dropout rate during the study.

This trial is registered with ClinicalTrials.gov, number NCT01416181.

\section{Role of the funding source}

The funder initiated and provided funding for this study and drafted and provided medical writing and editorial support in the development of this manuscript. The funder was also involved in study design, data collection, data analysis, and data interpretation, and reviewed and provided feedback on this manuscript. The authors, who include both employees of the funder and academic investigators, had full editorial control of this manuscript and provided final approval of all content. The corresponding author had full access to the study data and 
had final responsibility for the decision to submit for publication.

\section{Results}

Between Sept 13, 2011, and July 16, 2015, 889 patients were randomly assigned in part 1 , and 888 patients received at least one infusion of the study drug (natalizumab, $n=439$; placebo, $n=449$; figure 1). 887 patients were included in the part 1 intention-to-treat population (natalizumab, $n=439$; placebo, $n=448$ ). One placebo-treated patient received the first dose at week 28 and was therefore not included in the part 1 intention-to-treat population. 655 (74\%) patients (natalizumab, $n=336$; placebo, $n=319$ ) completed treatment through to week 96, and 638 (72\%) patients (natalizumab, $n=326$; placebo, $n=312$ ) completed the study through to the end of part 1 (week 108). Following part 1 , 566 patients continued into part 2 (continuing natalizumab, $\mathrm{n}=292$; initiating natalizumab, $\mathrm{n}=274$ ), and 565 patients received the study drug and were included in the part 2 intention-to-treat population (continuing natalizumab, $n=291$; initiating natalizumab, $n=274$ ). In part 2, the first treatment was received on Dec 2, 2013, and the study ended on April 13, 2016. The median total followup time, including the 96 weeks of part 1, was 157 weeks (range 108-221) for patients initiating natalizumab (switching from placebo) and 160 weeks (118-213) for patients continuing natalizumab.

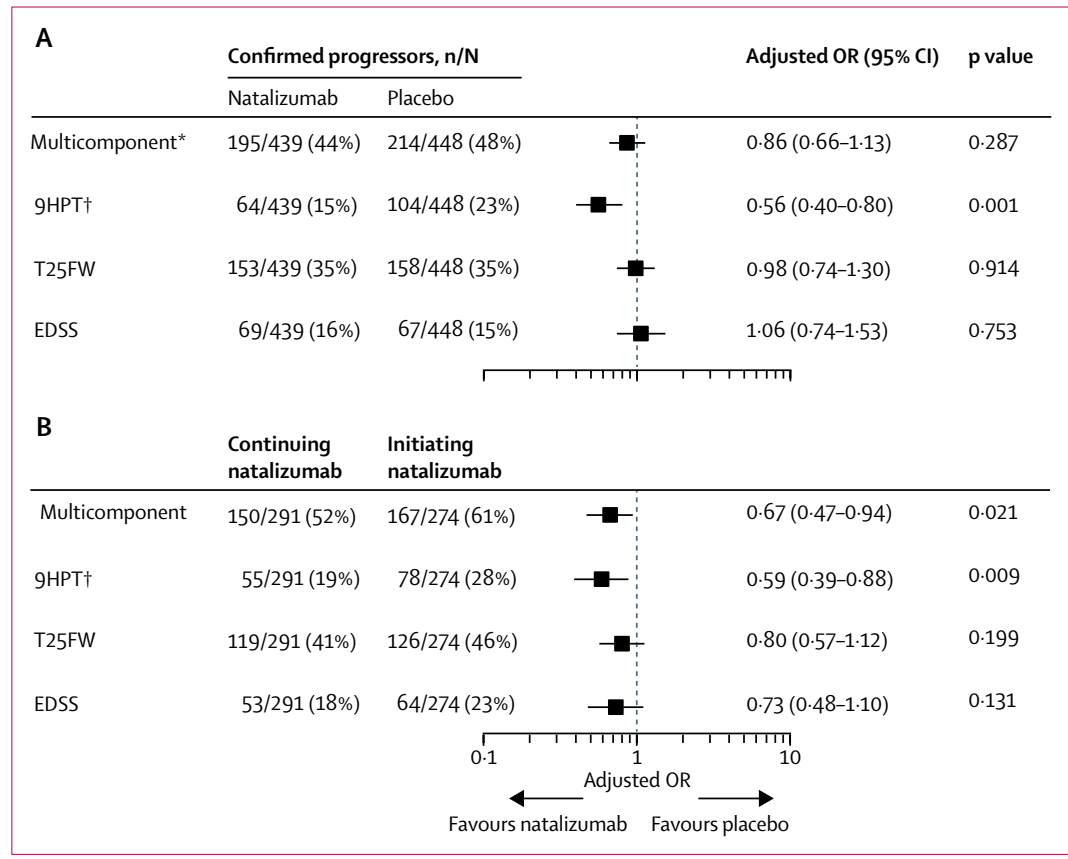

Figure 2: Proportion of patients with confirmed disability progression in the randomised, double-blind, placebo-controlled phase (part 1) and the open-label extension (part 2)

(A) Patients who entered the ASCEND study who showed confirmed disability progression over 96 weeks (part 1 intention-to-treat population). (B) Patients who entered the open-label extension of the ASCEND study who showed confirmed progression over 108 weeks, with confirmation up to 156 weeks (part 2 intention-to-treat population). $\mathrm{OR}=$ odds ratio. $9 \mathrm{HPT}=9$-Hole Peg Test. T25FW=Timed 25-Foot Walk. EDSS=Expanded Disability Status Scale. *Primary endpoint. †Either hand.
At baseline for part 1, clinical characteristics were balanced between treatment groups. Characteristics were also balanced between patients from part 1 and those who enrolled into part 2 (table 1). Baseline characteristics were generally similar between patients in the ASCEND part 1 intention-to-treat population who entered part 2 and those who did not (appendix). In the part 1 intention-to-treat population $(\mathrm{n}=887)$, the mean time since first multiple sclerosis symptoms was $16 \cdot 5$ (SD 7.7) years. At baseline, ambulatory impairment was present in all patients and was advanced in the majority; 556 (63\%) patients had EDSS scores of 6.0-6.5 (requiring walking aid) and only $13(1 \%)$ patients had an EDSS score of $3 \cdot 0(n=7$ for natalizumab, $\mathrm{n}=6$ for placebo). The overall mean baseline EDSS score was 5.6 (SD 0.9). As intended by the study design, enrolled patients with secondary progressive multiple sclerosis had minor relapse activity; 744 (84\%) were relapse-free within the year before baseline assessment $(\mathrm{n}=373$ for the natalizumab group, $\mathrm{n}=371$ for the placebo group) and 627 (71\%) were relapse-free within the 2 years before baseline assessment $(n=312$ for the natalizumab group, $\mathrm{n}=315$ for the placebo group).

ASCEND did not meet the primary endpoint for part 1 assessed at 2 years (figure 2 ). The proportion of confirmed progressors on the primary endpoint was lower in the natalizumab group (195 [44\%] of 439) than in the placebo group (214 [48\%] of 448), but the difference was not significant $(\mathrm{p}=0 \cdot 287)$. No treatment effect was observed on two components of the primary endpoint that measure progression of ambulatory disability: EDSS and T25FW (figure 2). However, natalizumab treatment was associated with a nominally significant $44 \%$ reduction in the relative risk of confirmed upper-limb disability progression as measured by $9 \mathrm{HPT}$, the third component of the primary endpoint (64 [15\%] with natalizumab vs 104 [23\%] with placebo; adjusted odds ratio [OR] 0.56 [95\% CI 0.40-0.80]; $\mathrm{p}=0 \cdot 001$; figure 2).

In part 1 , no significant differences between treatment groups in the change from baseline to year 2 were seen in any of the secondary endpoints, including MSWS-12, ABILHAND, and MSIS-29 (appendix). From week 24 to week 96 in part 1 , the mean percentage change in whole brain volume was $-0.66 \%$ (SD 0.60 ) in natalizumabtreated patients and $-0.72 \%(0.66)$ in placebo-treated patients $(\mathrm{p}=0 \cdot 242)$

Exploratory analyses suggested that the effect of natalizumab on slowing upper-limb disability accumulation as measured by 9HPT in part 1 was observed regardless of baseline $\mathrm{Gd}+$ lesions and was apparent in patients without relapses in the 1-2 years before the study (appendix), although differences between subgroups were not significant.

In additional exploratory analyses, natalizumab improved the ARR and outcomes of MRI endpoints compared with placebo (appendix).

In part 1, 410 (91\%) patients in the placebo group and $401(91 \%)$ in the natalizumab group reported one or more 
adverse events (table 2). Overall, adverse events were similar between natalizumab-treated and placebo-treated patients in part 1 (table 2). Natalizumab was generally well tolerated, with observed adverse events consistent with the known safety profile of natalizumab at the approved dose ( $300 \mathrm{mg}$ every 4 weeks). ${ }^{15}$ Table 3 shows all serious adverse events occurring in $1 \%$ or more patients in both treatment groups; all serious adverse events occurring in one or more patients are reported in the appendix. In part 1 , the proportion of patients reporting a serious adverse event was similar for the two treatment groups (90 [20\%] for natalizumab vs 100 [22\%] for placebo; table 3). Multiple sclerosis relapse was the most frequently reported serious adverse event in the two treatment groups, occurring in $21(5 \%)$ patients in the natalizumab group versus $28(6 \%)$ in the placebo group. The only other serious adverse events reported in $1 \%$ or more patients in either treatment group were urinary tract infection (five [1\%] in the natalizumab group vs 12 [3\%] in the placebo group), fall (six [1\%] vs three $[<1 \%]$ ), and pneumonia (two $[<1 \%]$ vs five [1\%]; table 3 ). During the course of part 1 , two patients receiving natalizumab died: one of lung cancer, and the other of septic shock with multiple organ failure. The investigators assessed both events to be unrelated to study treatment.

For the part 2 primary endpoint, 245 (84\%) patients continuing natalizumab and 250 (91\%) initiating natalizumab reported one or more adverse events (table 2). Adverse events were generally similar among patients continuing natalizumab and those initiating natalizumab in part 2 (table 2). No deaths occurred in part 2, and serious adverse events were reported by $39(13 \%)$ patients continuing natalizumab and 24 (9\%) initiating natalizumab (table 3 ). As in part 1, multiple sclerosis relapse was the most frequently reported serious adverse event, occurring in five $(2 \%)$ patients in each group. No cases of progressive multifocal leukoencephalopathy occurred in part 1 or 2 , and no new safety concerns were identified during the course of the study.

For secondary efficacy analyses in part 2, the proportion of patients who progressed on the multicomponent endpoint was lower in the natalizumabtreated group than in the placebo-treated group (150 [52\%] vs 167 [61\%]; adjusted OR 0.67 [95\% CI 0.47-0.94]; $\mathrm{p}=0.021$; figure 2). As in part 1 , upper-limb progression assessed by $9 \mathrm{HPT}$ in part 2 was lower in patients continuing natalizumab than in those initiating natalizumab (55 [19\%] vs 78 [28\%]; adjusted OR 0.59 [95\% CI 0.39-0.88]; $\mathrm{p}=0 \cdot 009$; figure 2). All other secondary endpoints in part 2 did not show a treatment effect. An exploratory analysis done with Kaplan-Meier estimates of the cumulative probability, over 156 weeks, of time to confirmed progression on the multicomponent endpoint (including the total part 1 intention-to-treat population, with data from patients who did not enrol in part 2 censored at week 108) showed significantly increased separation over time between patients originally

\begin{tabular}{|c|c|c|c|c|}
\hline & \multicolumn{2}{|l|}{ Part 1} & \multicolumn{2}{|l|}{ Part 2} \\
\hline & $\begin{array}{l}\text { Placebo } \\
(n=449)\end{array}$ & $\begin{array}{l}\text { Natalizumab } \\
300 \mathrm{mg} \\
(\mathrm{n}=439)\end{array}$ & $\begin{array}{l}\text { Initiating } \\
\text { natalizumab } \\
(\mathrm{n}=274)\end{array}$ & $\begin{array}{l}\text { Continuing } \\
\text { natalizumab } \\
(\mathrm{n}=291)\end{array}$ \\
\hline Patients with one or more events & $410(91 \%)$ & $401(91 \%)$ & 250 (91\%) & $245(84 \%)$ \\
\hline \multicolumn{5}{|c|}{ Events occurring in $10 \%$ or more patients in any group } \\
\hline Urinary tract infection & $107(24 \%)$ & $102(23 \%)$ & $56(20 \%)$ & $53(18 \%)$ \\
\hline Nasopharyngitis & $73(16 \%)$ & $98(22 \%)$ & $39(14 \%)$ & $47(16 \%)$ \\
\hline Fall & $86(19 \%)$ & $87(20 \%)$ & $38(14 \%)$ & $44(15 \%)$ \\
\hline Multiple sclerosis relapse & $122(27 \%)$ & $73(17 \%)$ & $34(12 \%)$ & $27(9 \%)$ \\
\hline Headache & $50(11 \%)$ & $66(15 \%)$ & $17(6 \%)$ & $26(9 \%)$ \\
\hline Fatigue & $53(12 \%)$ & $59(13 \%)$ & $35(13 \%)$ & $33(11 \%)$ \\
\hline Upper respiratory tract infection & $30(7 \%)$ & $48(11 \%)$ & $11(4 \%)$ & $10(3 \%)$ \\
\hline Back pain & $51(11 \%)$ & $46(10 \%)$ & $41(15 \%)$ & $35(12 \%)$ \\
\hline Arthralgia & $40(9 \%)$ & $43(10 \%)$ & $26(9 \%)$ & $20(7 \%)$ \\
\hline Pain in hands and feet & $42(9 \%)$ & $42(10 \%)$ & $28(10 \%)$ & $28(10 \%)$ \\
\hline Muscular weakness & $39(9 \%)$ & $28(6 \%)$ & $31(11 \%)$ & $15(5 \%)$ \\
\hline
\end{tabular}

\begin{tabular}{|c|c|c|c|c|}
\hline & \multicolumn{2}{|l|}{ Part 1} & \multicolumn{2}{|l|}{ Part 2} \\
\hline & $\begin{array}{l}\text { Placebo } \\
(n=449)\end{array}$ & $\begin{array}{l}\text { Natalizumab } \\
(\mathrm{n}=439)\end{array}$ & $\begin{array}{l}\text { Initiating } \\
\text { natalizumab } \\
(\mathrm{n}=274)\end{array}$ & $\begin{array}{l}\text { Continuing } \\
\text { natalizumab } \\
(\mathrm{n}=291)\end{array}$ \\
\hline Patients with one or more events & $100(22 \%)$ & $90(20 \%)$ & $24(9 \%)$ & $39(13 \%)$ \\
\hline \multicolumn{5}{|c|}{ Events occurring in $1 \%$ or more patients in any group } \\
\hline Multiple sclerosis relapse & $28(6 \%)$ & $21(5 \%)$ & $5(2 \%)$ & $5(2 \%)$ \\
\hline Urinary tract infection & $12(3 \%)$ & $5(1 \%)$ & $1(<1 \%)$ & $4(1 \%)$ \\
\hline Pneumonia & $5(1 \%)$ & $2(<1 \%)$ & $1(<1 \%)$ & 0 \\
\hline Multiple sclerosis & $5(1 \%)$ & 0 & 0 & 0 \\
\hline Fall & $3(<1 \%)$ & $6(1 \%)$ & $1(<1 \%)$ & $1(<1 \%)$ \\
\hline Urosepsis & $1(<1 \%)$ & $3(<1 \%)$ & $1(<1 \%)$ & $4(1 \%)$ \\
\hline
\end{tabular}

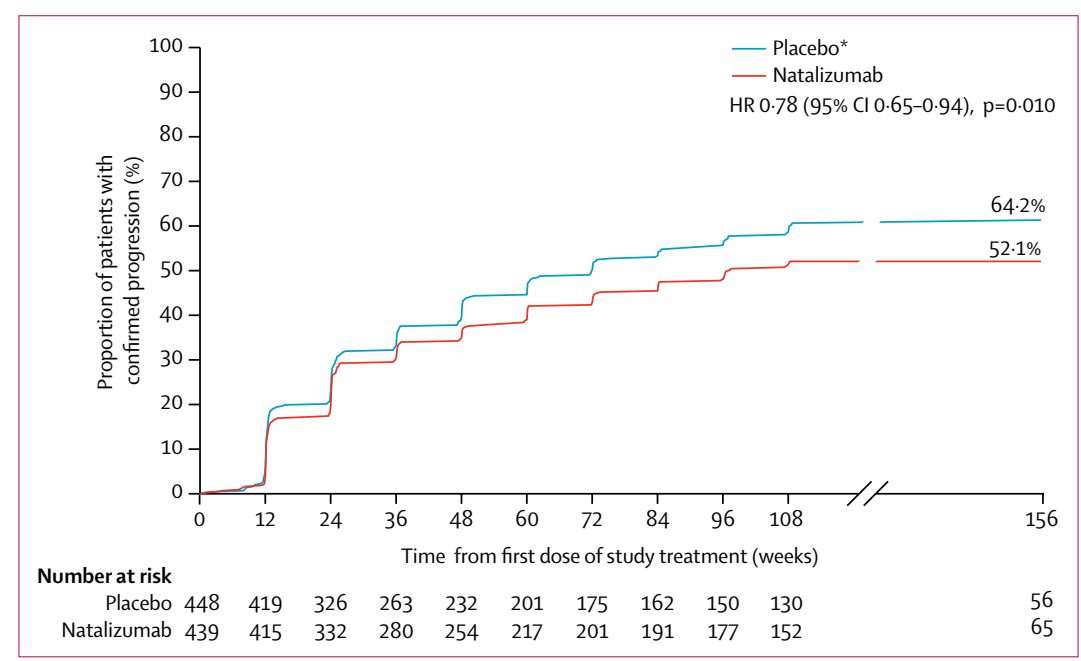

Figure 3: Time to confirmed disability progression on the multicomponent endpoint in parts 1 and 2 The part 1 intention-to-treat population contributed progression data over 84 weeks, and the part 2 intention-to-treat population additionally contributed progression data at weeks 96 and 108. *Includes patients taking placebo in part 1 and patients initiating natalizumab in part 2 . $H R=$ hazard ratio. 
randomly assigned to natalizumab and those randomly assigned to placebo who initiated natalizumab at week 108 $(52 \cdot 1 \%$ vs $64 \cdot 2 \%$; $\mathrm{p}=0 \cdot 010$; figure 3$)$.

MRI endpoints based on focal inflammation in part 2 are shown in the appendix. During part 2, the risk of relapse was $39.3 \%$ lower for patients continuing natalizumab than for those initiating natalizumab (ARR 0.11 vs 0.19 ; rate ratio 0.607 [95\% CI $0.424-0 \cdot 868$; $\mathrm{p}=0 \cdot 006)$.

\section{Discussion}

During the 2 year randomised treatment stage (part 1) of this phase 3 clinical trial, natalizumab did not significantly reduce disability progression as assessed by the primary multicomponent endpoint and secondary endpoints in patients with secondary progressive multiple sclerosis. However, progression of the upperlimb component of the primary disability endpoint, as assessed by 9HPT, was reduced in both parts 1 and 2 . Significant efficacy was also observed on reductions in ARR and MRI measures of focal inflammation, as expected from previous trials in patients with relapsing remitting multiple sclerosis. ${ }^{15}$ Imaging studies showed that treatment did not affect loss of whole brain volume during part 1 , but that whole brain volume loss was significantly lower when considering parts 1 and 2 together or part 2 separately.

Interpretation of the positive treatment effects in part 2 is limited by the fact that this was an open-label extension phase of ASCEND and that the study was not designed with part 1 as an interim analysis. Significance was not achieved on the primary endpoint during part 1; therefore, significance in part 2 is only nominal, since adjustments for multiplicity were not applied. Furthermore, because of early termination, part 2 did not last for the planned 2 years beyond the end of part 1 . This early closure might have precluded detection of outcomes requiring a longer period of time to show a treatment effect. It should also be noted that, for part 1 , disability progression was required to be confirmed not only at a visit 6 or more months later, with the period of 3 months following onset of an adjudicated clinical relapse excluded, but also at week 96. This is possibly the most stringent requirement for verification of progression adopted for progressive multiple sclerosis trials to date. During part 1, the attrition rate was somewhat higher than expected (638 [72\%] of 888 patients completed 108 weeks, so the dropout rate [28\%] was higher than the predicted dropout of $20 \%$ ). However, the numbers of patients in each treatment group who completed the study were similar ( $\mathrm{n}=326$ for natalizumab vs $\mathrm{n}=312$ for placebo). Additionally, it was prespecified that possible disability progression for patients who discontinued could be confirmed on the basis of the discontinuation visit measurements, and progression could also be considered confirmed if patients discontinued treatment because of inadequate efficacy (at the discretion of the investigator). The effect of attrition on the validity of the conclusions is therefore limited. Patients in part 2 were a subset of the original randomised population, and disability measurements for those not continuing to part 2 were incomplete because of early termination of that phase of the trial, so selection bias could have occurred. However, patients who did or did not enter the open-label extension had similar baseline characteristics and disability progression outcomes in part 1 , suggesting that selection bias was minimal. Bias was limited further by maintaining full masking to the initial treatment allocation through the open-label extension. Masking was also maintained for the imaging measurements, although bias might have occurred because of pseudoatrophy affecting whole brain matter volume in patients newly initiating natalizumab in part 2.

In contrast to several previous trials in secondary progressive multiple sclerosis, ${ }^{2,10,11}$ ASCEND enrolled patients with predominantly non-relapsing secondary progressive multiple sclerosis, who constitute a multiple sclerosis population with high unmet needs. Furthermore, at baseline, the majority of ASCEND patients had advanced disability with ongoing disease progression unrelated to relapses: $63 \%$ had EDSS scores of $6 \cdot 0-6 \cdot 5$, and $71 \%$ had no relapses in the 2 years before baseline assessment. The on-study ARR in patients randomly assigned to placebo was low $(0 \cdot 17$ [95\% CI $0 \cdot 14-0 \cdot 21]$ appendix), further supporting the low relapsing activity of the enrolled population. These patients represent the secondary progressive multiple sclerosis population for whom no effective therapy is available to delay or prevent further disability progression unrelated to relapses.

Detecting change with available validated outcome measures in a slowly progressing multiple sclerosis population over a short period of observation is difficult. The multicomponent endpoint used in this study might be more sensitive to changes in disability than the EDSS alone, ${ }^{22}$ but two of its three components focus on lowerlimb function and were not affected by treatment. The EDSS, in particular, has been shown to have poor responsiveness to disease progression and treatment effects in patients with high baseline scores. ${ }^{21}$ Other studies in patients with secondary progressive multiple sclerosis have used composite outcome measurements and reported differences in outcomes assessing upperlimb versus lower-limb function, ${ }^{11,24}$ highlighting the importance of using sensitive measures of disease worsening in secondary progressive multiple sclerosis trials that include key domains of function in addition to ambulation..$^{25}$

In part 1 of this study, we observed an apparent beneficial effect of natalizumab on limb function but not on measures of ambulation. Although this difference could be explained by different treatment effects on neural systems serving different functions, the findings are also consistent with the possibility that the benefits of stopping inflammation from further damaging the CNS 
manifest after a delay that is shorter in pathways with shorter axons than in pathways with longer axons. ${ }^{26}$ Axons undergoing injury might continue to degenerate even after the mechanisms of injury are inhibited by treatment (eg, from trans-synaptic degeneration or because demyelination renders them vulnerable to injury). Delayed clinical and imaging responses have been observed in trials of interferon beta and natalizumab, ${ }^{26-28}$ and are apparent in the results of the open-label phase of the ASCEND trial. Residual degeneration should abate more quickly in shorter axon pathways than in longer ones because shorter axons have a lower lesion burden. If the resulting length-dependent therapeutic lag does exist, future trial designs might need to consider longer treatment phases to capture benefits in all key aspects of disability and to plan for domain-specific outcome measures, which will respond at successively later stages in the trial.

In general, the delay in appearance of any treatment effects in patients with secondary progressive multiple sclerosis differs substantially from the rapid onset of the effects of natalizumab in patients with relapsingremitting multiple sclerosis, ${ }^{29}$ suggesting a difference in the underlying disease mechanisms of relapsingremitting and secondary progressive multiple sclerosis with regard to their response to $\alpha 4$ integrin antagonism. The observation that the beneficial effects of natalizumab on upper-limb disability progression occurred regardless of baseline $\mathrm{Gd}+$ lesions or relapses before entry further suggests that treatment effects in secondary progressive multiple sclerosis might occur through different mechanisms to those observed in relapsing-remitting multiple sclerosis.

Natalizumab was generally well tolerated throughout both parts of ASCEND. No new or unexpected safety concerns were associated with its use in a secondary progressive multiple sclerosis population, and the observed safety profile was consistent with that of previous studies of natalizumab in patients with relapsing-remitting multiple sclerosis. ${ }^{15}$ The beneficial effects of natalizumab on ARR and on new and active brain lesions in ASCEND were also consistent with previous findings in patients with relapsing multiple sclerosis ${ }^{15}$ and with continued anti-inflammatory activity in secondary progressive multiple sclerosis. ${ }^{14}$ However, natalizumab is not approved for treatment of patients with non-relapsing secondary progressive multiple sclerosis, and its benefit-risk profile has only been examined for treatment of patients with relapsing forms of multiple sclerosis.

The results of the 9HPT analyses consistently suggest that natalizumab delays loss of upper-limb function, and exploratory analyses suggest that this treatment effect occurred independently of active brain lesions (appendix). The $20 \%$ threshold of change in the 9HPT used in the primary endpoint in this study is consistent with that of previous work, which indicates that this difference corresponds to a clinically meaningful change in upperlimb function. ${ }^{30}$ Upper-limb function correlates independently with quality of life $\mathrm{e}^{31}$ and is a strong predictor of treatment costs in multiple sclerosis. ${ }^{32}$ Preservation of upper-limb function ranks highly among treatment benefits desired by disabled patients with multiple sclerosis, in some instances more highly than lower-limb function for maintenance of independence. Patients also consider that those confined to wheelchairs should not be excluded from future clinical trials in progressive multiple sclerosis. ${ }^{33}$ Treatments that can preserve ability in a single functional domain could, therefore, still be valuable for patients with secondary progressive multiple sclerosis in real-world settings.

\section{Contributors}

DLA, MSF, MDG, H-PH, EKH, DJ, RK, AM, FS, DC, AD, DM, and DS contributed to study design. DC, DS, and BY administered the study. DLA, MSF, MDG, H-PH, EKH, DJ, RK, AM, FS, DC, AD, DM, and DS contributed to data collection. IC, FF, and NL did the data analysis. DLA, MS, MDG, H-PH, EKH, DJ, RK, AM, FS, DC, NC, IC, $\mathrm{AD}, \mathrm{FF}, \mathrm{NL}, \mathrm{DM}, \mathrm{DS}, \mathrm{BY}$, and P-RH contributed to interpretation of the data. All authors read and approved the final manuscript.

\section{ASCEND investigators group}

Belgium: Emmanuel Bartholomé, Marie D’Hooghe, Massimo Pandolfo, Bart Van Wijmeersch. Canada: Virender Bhan, Gregg Blevins, Donald Brunet, Virginia Devonshire, Pierre Duquette, Mark Freedman, François Grand'Maison, François Jacques, Yves Lapierre, Liesly Lee, Sarah Morrow, Michael Yeung. Czech Republic: Michal Dufek, Eva Kubala Havrdová, Petr Kanovsky, Ivana Stetkarova, Marika Talabova. Denmark: Jette Frederiksen, Matthias Kant, Thor Petersen, Mads Ravnborg, Finn Sellebjerg. Finland: Laura Airas, Irina Elovaara, Juha-Pekka Eralinna, Taneli Sarasoja. France: Abdullatif Al Khedr, David Brassat, Bruno Brochet, William Camu, Marc Debouverie, David Laplaud, Christine Lebrun Frenay, Jean Pelletier, Patrick Vermersch, Sandra Vukusic. Germany: Karl Baum, Achim Berthele, Juergen Faiss, Peter Flachenecker, Reinhard Hohlfeld, Markus Krumbholz, Christoph Lassek, Mathias Maeurer, Sven Meuth, Tjalf Ziemssen. Ireland: Orla Hardiman, Christopher McGuigan. Israel: Anat Achiron, Dimitrios Karussis. Italy: Roberto Bergamaschi, Vincenzo Brescia Morra, Giancarlo Comi, Salvatore Cottone, Luigi Grimaldi,

Giovanni Luigi Mancardi, Luca Massacesi, Ugo Nocentini, Marco Salvetti, Elio Scarpini, Patrizia Sola, Gioacchino Tedeschi, Maria Trojano, Mauro Zaffaroni. Netherlands: Stephan Frequin, Raymond Hupperts, Joep Killestein, Hans Schrijver, Ronald Van Dijl, Erik van Munster. Poland: Maciej Czarnecki, Wieslaw Drozdowski, Waldemar Fryze, Hanka Hertmanowska, Jan Ilkowski, Anna Kaminska, Gabriela Klodowska-Duda, Maciej Maciejowski, Ewa Motta, Ryszard Podemski, Andrzej Potemkowski, Teresa Rog, Krzysztof Selmaj, Zbigniew Stelmasiak, Adam Stepien, Andrzej Tutaj, Jacek Zaborski. Russia: Alexey Boyko, Zanna Chefranova, Evgeny Evdoshenko, Farit Khabirov, Stella Sivertseva, Eduard Yakupov.

Spain: Jose Carlos Alvarez Cermeño, Antonio Escartin Oscar Fernandez Fernandez, Antonio Garcia-Merino, Miguel Angel Hernandez Perez, Guillermo Izquierdo Ayuso, José Meca Lallana, Xavier Montalban Gairin, Celia Oreja-Guevara, Albert Saiz Hinarejos. Sweden: Martin Gunnarsson, Jan Lycke, Claes Martin, Fredrik Piehl, Homayoun Roshanisefat, Peter Sundstrom UK: Martin Duddy, Bruno Gran, Timothy Harrower, Jeremy Hobart, Raju Kapoor, Martin Lee, Paul Mattison, Richard Nicholas, Owen Pearson, Waqar Rashid, David Rog, Basil Sharrack, Eli Silber, Ben Turner, Anna Williams, John Woolmore, Carolyn Young. USA: Daniel Bandari, Joseph Berger, Ann Camac, Stanley Cohan, Jill Conway, Keith Edwards, Michelle Fabian, Jack Florin, Steven Freedman, Dennis Garwacki, Myla Goldman, Daniel Harrison, Craig Herrman, Deren Huang, Adil Javed, Douglas Jeffery, Stephen Kamin, George Katsamakis, Bhupendra Khatri, Annette Langer-Gould, Sharon Lynch, David Mattson, Tamara Miller, Augusto Miravalle. Harold Moses, Suraj Muley, 
James Napier, Allen Nielsen, Andrew Pachner, Gabriel Pardo,

MaryAnn Picone, Derrick Robertson, Walter Royal, Christopher Sheppard, Ben Thrower, Cary Twyman, Emmanuelle Waubant, Jeanette Wendt, Vijayshree Yadav, Rana Zabad, Greg Zarelli.

\section{Declaration of interests}

RK has received support from the NIHR Biomedical Research Centre at University College London Hospitals NHS Foundation Trust and University College London (UCLH/UCL) and personal compensation for consultancies, lectures, participation in advisory boards, and support for travel to medical meetings from Biogen, Genzyme, Karo Bio, Novartis, Roche, and Teva. UCL Hospitals received payment for investigation and follow-up of participants in the ASCEND trial. P-RH, NC, IC, AD, FF, NL, BY, and DS are employees of and hold stock or stock options, or both, in Biogen. DLA has served on advisory boards, received speaker honoraria, served as a consultant for, or received research support from, Adelphi, Biogen, Genentech, MedDay, Novartis Pharma AG, Pfizer, Receptos/Celgene, Roche, and Sanofi, and holds stock in NeuroRx Research. MSF has received honoraria or consulting fees from Actelion, Bayer HealthCare, Clene Nanomedicine, Hoffman-La Roche, Merck Serono, Sanofi, Genzyme, and Teva Canada Innovation; participated in company advisory boards, steering committees, or boards of directors for Actelion, Bayer HealthCare, Chugai, EMD Serono, and Sanofi Genzyme; received travel support from Biogen; and received grants for research from Sanofi Genzyme. MDG has received personal compensation for consulting services from Acorda Therapeutics, Adamas, AtlasD, EMD Serono, Endece, Genzyme, Novartis, and Serepta; honorarium from the American Academy of Neurology; and research support from Biogen, Novartis Pharmaceuticals, NMSS, and NINDS. H-PH has received personal compensation for consulting services and speaking at scientific symposia from Biogen, GeNeuro, Genzyme, Merck Serono, Novartis, Octapharma, Opexa, Roche, and Teva. EKH has received research support from Biogen and The Czech Ministry of Education (project PROGRESQ27/LF1) and honoraria from Actelion, Biogen, Celgene, Merck Serono, Novartis, Roche, Sanofi Genzyme, and Teva. DJ has received research funding from Biogen, Genentech, and Novartis, and personal compensation for speaking or consulting services from Acorda, Bayer, Biogen, Genentech, Mallinkrodt, Novartis, Sanofi Genzyme, Serono, and Teva. AM has received research funding from Biogen, Genentech, Genzyme, Novartis, Roche, and Sanofi and personal compensation for consulting services from Accordant Health Services, Adamas, Biogen, Celgene, Genentech, Mallinkrodt, Mapi-Pharma, Novartis, and Roche. FS has served on scientific advisory boards, on steering committees of clinical trials, or as a consultant for, or received support for, congress participation, speaker honoraria, or research support for his laboratory from Biogen, Genzyme, Merck Serono, Novartis, and Sanofi. DC was an employee of Biogen at the time of this analysis and is now an employee of Fulcrum Therapeutics. DM was an employee of Biogen at the time of this analysis and is now an employee of Amgen. Fulcrum Therapeutics, and Amgen were not in any way associated with this study.

\section{Acknowledgments}

Diogo Amarante (Biogen, Cambridge, MA), who contributed substantially to the acquisition, analysis, and interpretation of ASCEND data, passed away before development of this manuscript and therefore cannot qualify for authorship as per International Committee of Medical Journal Editors (ICMJE) criteria. We gratefully acknowledge his contributions to this manuscript, as well as the contributions of Thorsten Eickenhorst, a medical director for the study, who passed away before the development of this manuscript. All named authors meet the ICMJE criteria for authorship for this manuscript and take responsibility for the integrity of the work as a whole. Biogen provided funding for medical writing support in the development of this manuscript; Alexandra W Davis (Ashfield Healthcare Communications, Middletown, CT, USA) wrote the first draft of this manuscript based on input from authors, and Joshua Safran (Ashfield Healthcare Communications) copyedited and styled the manuscript as per journal requirements. Biogen reviewed and provided feedback on the manuscript to the authors. The authors had full editorial control of the manuscript and provided their final approval of all content.
References

1 Lublin FD, Reingold SC, Cohen JA, et al. Defining the clinical course of multiple sclerosis: the 2013 revisions. Neurology 2014; 83: 278-86.

2 The North American Study Group on Interferon beta-1b in Secondary Progressive MS. Interferon beta-1b in secondary progressive MS: results from a 3-year controlled study. Neurology 2004; 63: 1788-95.

3 Lublin F, Miller DH, Freedman MS, et al. Oral fingolimod in primary progressive multiple sclerosis (INFORMS): a phase 3, randomised, double-blind, placebo-controlled trial. Lancet 2016; 387: 1075-84.

4 Wolinsky JS, Narayana PA, O'Connor P, et al. Glatiramer acetate in primary progressive multiple sclerosis: results of a multinational, multicenter, double-blind, placebo-controlled trial. Ann Neurol 2007; 61: 14-24.

5 Secondary Progressive Efficacy Clinical Trial of Recombinant Interferon-Beta-1a in MS (SPECTRIMS) Study Group. Randomized controlled trial of interferon- beta-1a in secondary progressive MS: clinical results. Neurology 2001; 56: 1496-504.

6 Pohlau D, Przuntek H, Sailer M, et al. Intravenous immunoglobulin in primary and secondary chronic progressive multiple sclerosis: a randomized placebo controlled multicentre study. Mult Scler 2007; 13: 1107-17.

7 Chataway J, Schuerer N, Alsanousi A, et al. Effect of high-dose simvastatin on brain atrophy and disability in secondary progressive multiple sclerosis (MS-STAT): a randomised, placebo-controlled, phase 2 trial. Lancet 2014; 383: 2213-21.

8 Tourbah A, Lebrun-Frenay C, Edan G, et al. MD1003 (high-dose biotin) for the treatment of progressive multiple sclerosis: a randomised, double-blind, placebo-controlled study. Mult Scler 2016; 22: 1719-31.

9 Montalban X, Hauser SL, Kappos L, et al. Ocrelizumab versus placebo in primary progressive multiple sclerosis. N Engl J Med 2017; 376: 209-20.

10 European Study Group on interferon beta- $1 \mathrm{~b}$ in secondary progressive MS. Placebo-controlled multicentre randomised trial of interferon $\beta$-1b in treatment of secondary progressive multiple sclerosis. Lancet 1998; 352: 1491-97.

11 Hartung HP, Gonsette R, Konig N, et al. Mitoxantrone in progressive multiple sclerosis: a placebo-controlled, double-blind, randomised, multicentre trial. Lancet 2002; 360: 2018-25.

12 Freedman MS, Bar-Or A, Oger J, et al. A phase III study evaluating the efficacy and safety of MBP8298 in secondary progressive MS. Neurology 2011; 77: 1551-60.

13 Kappos L, Bar-Or A, Cree B, et al. Efficacy of siponimod in secondary progressive multiple sclerosis: results of the phase 3 study. Neurology 2017; 88 (suppl 16): CT.002.

14 Sellebjerg F, Cadavid D, Steiner D, Villar LM, Reynolds R, Mikol D. Exploring potential mechanisms of action of natalizumab in secondary progressive multiple sclerosis. Ther Adv Neurol Disord 2016; 9: 31-43.

15 Polman CH, O'Connor PW, Havrdova E, et al. A randomized, placebo-controlled trial of natalizumab for relapsing multiple sclerosis. N Engl J Med 2006; 354: 899-910.

16 Yednock TA, Cannon C, Fritz LC, Sanchez-Madrid F, Steinman L, Karin N. Prevention of experimental autoimmune encephalomyelitis by antibodies against alpha 4 beta 1 integrin. Nature 1992; 356: 63-66.

17 Rudick RA, Sandrock A. Natalizumab: alpha 4-integrin antagonist selective adhesion molecule inhibitors for MS. Expert Rev Neurother 2004; 4: 571-80.

18 Khademi M, Bornsen L, Rafatnia F, et al. The effects of natalizumab on inflammatory mediators in multiple sclerosis: prospects for treatment-sensitive biomarkers. Eur J Neurol 2009, 16: 528-36.

19 Romme Christensen J, Ratzer R, Bornsen L, et al. Natalizumab in progressive MS: results of an open-label, phase 2A, proof-of-concept trial. Neurology 2014; 82: 1499-507.

20 Cadavid D, Jurgensen S, Lee S. Impact of natalizumab on ambulatory improvement in secondary progressive and disabled relapsing-remitting multiple sclerosis. PLoS One 2013; 8: e53297.

21 Cadavid D, Tang Y, O'Neill G. Responsiveness of the Expanded Disability Status Scale (EDSS) to disease progression and therapeutic intervention in progressive forms of multiple sclerosis. Rev Neurol 2010; 51: 321-29 (in Spanish). 
22 Cadavid D, Cohen JA, Freedman MS, et al. The EDSS-Plus, an improved endpoint for disability progression in secondary progressive multiple sclerosis. Mult Scler 2017; 23: 94-105.

23 Barrett LE, Cano SJ, Zajicek JP, Hobart JC. Can the ABILHAND handle manual ability in MS? Mult Scler 2013; 19: 806-15.

24 Goodkin DE, Rudick RA, VanderBrug Medendorp S, et al. Low-dose $(7.5 \mathrm{mg})$ oral methotrexate reduces the rate of progression in chronic progressive multiple sclerosis. Ann Neurol 1995; 37: 30-40.

25 LaRocca NG, Hudson LD, Rudick R, et al. The MSOAC approach to developing performance outcomes to measure and monitor multiple sclerosis disability. Mult Scler 2017; published online Aug 11. DOI:10.1177/1352458517723718.

26 Giovannoni G, Cutter G, Sormani MP, et al. Is multiple sclerosis a length-dependent central axonopathy? The case for therapeutic lag and the asynchronous progressive MS hypotheses. Mult Scler Relat Disord 2017; 12: 70-78.

27 Tur C, Montalban X, Tintore M, et al. Interferon beta-1b for the treatment of primary progressive multiple sclerosis: five-year clinical trial follow-up. Arch Neurol 2011; 68: 1421-27.
28 Sastre-Garriga J, Tur C, Pareto D, et al. Brain atrophy in natalizumab-treated patients: a 3-year follow-up. Mult Scler 2015; 21: $749-56$

29 Kappos L, O'Connor PW, Polman CH, et al. Clinical effects of natalizumab on multiple sclerosis appear early in treatment course. J Neurol 2013; 260: 1388-95.

30 Feys P, Lamers I, Francis G, et al. The Nine-Hole Peg Test as a manual dexterity performance measure for multiple sclerosis. Mult Scler 2017; 23: 711-20.

31 Yozbatiran N, Baskurt F, Baskurt Z, Ozakbas S, Idiman E. Motor assessment of upper extremity function and its relation with fatigue, cognitive function and quality of life in multiple sclerosis patients. J Neurol Sci 2006; 246: 117-22.

32 Koch MW, Murray TJ, Fisk J, et al. Hand dexterity and direct disease related cost in multiple sclerosis. J Neurol Sci 2014; 341: 51-54.

33 Thompson A, Giovannoni G, Marta S, et al. Importance of upper limb function in advanced multiple sclerosis. Mult Scler 2016; 22 (suppl 3): 676.P1277. 